OPEN ACCESS

Edited by:

Hiroyasu Ejiri,

Research Center for Nuclear Physics,

Osaka University, Japan

Reviewed by:

Bhupal Dev,

Washington University in St. Louis,

United States

J.D. Vergados,

University of loannina, Greece

Javier Menendez,

University of Barcelona, Spain

*Correspondence:

Yoritaka Iwata

iwata_phys@08.alumni.u-

tokyo.ac.jp

Specialty section:

This article was submitted to

High-Energy and Astroparticle

Physics,

a section of the journal

Frontiers in Astronomy and Space

Sciences

Received: 20 June 2021

Accepted: 30 August 2021

Published: 19 November 2021

Citation:

Iwata Y and Sarkar S (2021) Interacting

Shell Model Calculations for

Neutrinoless Double Beta Decay of

${ }^{82}$ Se With Left-Right Weak

Boson Exchange.

Front. Astron. Space Sci. 8:727880.

doi: 10.3389/fspas.2021.727880

\section{Interacting Shell Model Calculations for Neutrinoless Double Beta Decay of ${ }^{82}$ Se With Left-Right Weak Boson Exchange}

\author{
Yoritaka Iwata ${ }^{1 *}$ and Shahariar Sarkar ${ }^{2}$ \\ ${ }^{1}$ Faculty of Chemistry, Materials and Bioengineering, Kansai University, Osaka, Japan, ${ }^{2}$ Indian Institute of Technology Ropar, \\ Rupnagar, India
}

In the present work, the $\lambda$ mechanism (left-right weak boson exchange) and the light neutrino-exchange mechanism of neutrinoless double beta decay is studied. In particular, much attention is paid to the calculation of nuclear matrix elements for one of the neutrinoless double beta decaying isotopes ${ }^{82} \mathrm{Se}$. The interacting shell model framework is used to calculate the nuclear matrix element. The widely used closure approximation is adopted. The higher-order effect of the pseudoscalar term of nucleon current is also included in some of the nuclear matrix elements that result in larger Gamow-Teller matrix elements for the $\lambda$ mechanism. Bounds on Majorana neutrino mass and lepton number violating parameters are also derived using the calculated nuclear matrix elements.

\footnotetext{
Keywords: neutrinoless double beta decay, $\lambda$ mechanism, nuclear shell model, nuclear matrix element, right-handed weak boson
}

\section{INTRODUCTION}

Neutrinoless double beta decay $(0 \nu \beta \beta)$ is a rare second-order weak nuclear process. In this process, neutrino comes as a virtual intermediate particle when two neutron pairs decay into two proton pairs inside some even-even nuclei. Thus, it violates the lepton number by two units. The $0 \nu \beta \beta$ experiment is one of the possible ways to determine the effective neutrino mass (Schechter and Valle, 1982; Tomoda, 1991; Avignone et al., 2008; Rodejohann, 2011; Deppisch et al., 2012) and can help to solve many mysteries of neutrinos, such as whether neutrinos are their own anti-particle (Majorana neutrino) or not (Dirac neutrino) (Schechter and Valle, 1982; Rodejohann, 2011; Deppisch et al., 2012).

As lepton number conservation is not exact in most of the beyond the standard model (BSM) physics theories, many particle mechanisms of $0 \nu \beta \beta$ have been proposed in different BSM theories such as light neutrino-exchange mechanism (Šimkovic et al., 1999; Rodin et al., 2006), heavy neutrino-exchange mechanism (Vergados et al., 2012), left-right symmetric mechanism (Mohapatra and Senjanović, 1980; Mohapatra and Vergados, 1981), and the supersymmetric particles exchange mechanism (Mohapatra, 1986; Vergados, 1987).

The decay rate for any particle mechanism of $0 \nu \beta \beta$ is connected by nuclear matrix elements (NMEs) and absolute neutrino mass. These NMEs are calculated using theoretical nuclear manybody models (Engel and Menéndez, 2017). Popular nuclear models are quasiparticle random phase approximation (QRPA) (Vergados et al., 2012), the interacting shell-model (ISM) (Caurier et al., 2008; Horoi and Stoica, 2010; Sen'kov and Horoi, 2013; Brown et al., 2014; Iwata et al., 2016), 
the interacting boson model (IBM) (Barea and Iachello, 2009; Barea et al., 2012), the generator coordinate method (GCM) (Rodríguez and Martínez-Pinedo, 2010), the energy density functional (EDF) theory (Rodríguez and Martínez-Pinedo, 2010; Song et al., 2014), and the projected Hartree-Fock Bogolibov model (PHFB) (Rath et al., 2010). Other techniques includes, $a b$ initio calculations for lower mass nuclei ( $\mathrm{A}=6-12)$ using variational Monte Carlo (VMC) method (Pastore et al., 2018; Cirigliano et al., 2019; Wang et al., 2019).

In the present work, we focus on the left-right weak boson $\left(W_{L^{-}}\right.$ $W_{R}$ ) exchange $\lambda$ mechanism along with the standard light neutrinoexchange mechanism $\left(W_{L}-W_{L}\right.$ exchange) of the $0 \nu \beta \beta$ mediated by light neutrinos (Bhupal Dev et al., 2015; Horoi and Neacsu, 2016; Šimkovic et al., 2017). The $\lambda$ mechanism has origin in the left-right symmetric mechanism with right-handed gauge boson at the $\mathrm{TeV}$ scale (Šimkovic et al., 2017). Thus, it will be interesting to study how the $\lambda$ mechanism can compete with the standard light neutrinoexchange mechanism when both the mechanisms co-exist. Hence, in the present work, we are eager to study the $\lambda$ and light neutrinoexchange mechanisms together.

In left-right symmetric model, there is another mass independent mechanism called $\eta$ mechanism which occurs through $W_{L}-W_{R}$ mixing. It will be interesting to study $\eta$ mechanism along with $\lambda$ mechanism of $0 v \beta \beta$. But, $\eta$ mechanism is suppressed due to $W_{L}-W_{R}$ mixing as compared to $\lambda$ mechanism (Barry and Rodejohann, 2013). Hence, in the present work, we are interested to study the mass independent $\lambda$ mechanism along with the mass dependent standard light neutrino-exchange mechanism. In future studies, we will extensively explore the $\eta$ mechanism of $0 \nu \beta \beta$ along with other mass independent and dependent mechanisms in left-right symmetric model.

One of the motivations of the present work is to include effects of some of the revisited formalism of Ref. (Štefánik et al., 2015) on light neutrino-exchange and $\lambda$ mechanism of $0 \nu \beta \beta$. The revised formalism was exploited to include the effects of the pseudoscalar term of nucleon currents. Using the revised formalism of Ref. (Štefánik et al., 2015), the NMEs for $\lambda$, and light neutrino-exchange mechanisms of $0 \nu \beta \beta$ are calculated using the QRPA model for several $0 v \beta \beta$ decaying isotopes using closure approximation in Ref. (Šimkovic et al., 2017). Most of the NMEs relevant for $\lambda$ and light neutrino-exchange mechanisms are also calculated using ISM in Ref. (Horoi and Neacsu, 2018) using the closure approximation for different $0 \nu \beta \beta$ decaying isotopes (including ${ }^{82} \mathrm{Se}$ ). In this case, some of the NMEs are calculated without including the higher-order terms (for example, pseudoscalar and weak magnetism terms) of the nucleon currents. Recently, using the revised formalism of Ref. (Štefánik et al., 2015), we have also calculated the NMEs for ${ }^{48} \mathrm{Ca}$ in Ref. (Sarkar et al., 2020a) using the non-closure approximation and found a significant change in some of the NMEs for including the pseudoscalar term. Thus, we have tried here to include the revised higher-order effect of the pseudoscalar term of nucleon current for the $\lambda$ mechanism of $0 \nu \beta \beta$ of ${ }^{82} \mathrm{Se}$ using ISM. The $0 \nu \beta \beta$ of ${ }^{82} \mathrm{Se}$ is one of the experimental interests of CUPID (Dolinski et al., 2019;
Pagnanini et al., 2019) and NEMO-3 (Arnold et al., 2020) experiments. Hence, it is important to study the nuclear structure aspects of $0 \nu \beta \beta$ of ${ }^{82} \mathrm{Se}$ theoretically. In recent years, one of the most important studies on light neutrinoexchange $0 \nu \beta \beta$ of ${ }^{82}$ Se was performed in the ISM framework in Ref. (Sen'kov et al., 2014) using the non-closure approximation. Here we focus on the $\lambda$ mechanism of $0 \nu \beta \beta$ of ${ }^{82} \mathrm{Se}$ in the closure approximation using the revised nucleon current term.

This paper is organized as follows. In Section 2, the expression for decay rate and the theoretical formalism to calculate NMEs for the $\lambda$ and light neutrino-exchange mechanisms of $0 \nu \beta \beta$ are presented. The results and discussion are presented in Section 3. A summary of the work is given in Section 4.

\section{THEORETICAL FRAMEWORK}

\subsection{Decay Rate for $\lambda$ Mechanism of $0 v \beta \beta$}

If both light neutrino-exchange $\left(W_{L}-W_{L}\right.$ exchange) and $\lambda$ mechanisms $\left(W_{L}-W_{R}\right.$ exchange) of $0 v \beta \beta$ co-exist, one can write the decay rate for $0 \nu \beta \beta$ as (Štefánik et al., 2015; Šimkovic et al., 2017)

$$
\left[T_{1 / 2}^{0 \nu}\right]^{-1}=\eta_{\nu}^{2} C_{m m}+\eta_{\lambda}^{2} C_{\lambda \lambda}+\eta_{\nu} \eta_{\lambda} \cos \psi C_{m \lambda},
$$

where the coupling constant $\lambda$ is defined as (Šimkovic et al., 2017)

$$
\lambda=\left(M_{W_{L}} / M_{W_{R}}\right)^{2} .
$$

The $M_{W_{L}}$ and $M_{W_{R}}$ are masses of the Standard Model lefthanded $W_{L}$ and right-handed $W_{R}$ gauge bosons, respectively. The $\eta_{\nu}$ of Eq. 1 is an effective lepton number violating parameters for $W_{L}-W_{L}$ exchange, $\eta_{\lambda}$ is an effective lepton number violating parameters for $W_{L}-W_{R}$ exchange, and $\psi$ denotes the $\mathrm{CP}$ violating phase. These parameters are given in Ref. (Šimkovic et al., 2017) as

$$
\begin{gathered}
\eta_{v}=\frac{m_{\beta \beta}}{m_{e}}, \quad \eta_{\lambda}=\lambda\left|\sum_{j=1}^{3} m_{j} U_{e j} T_{e j}^{*}\right|, \\
\psi=\arg \left[\left(\sum_{j=1}^{3} m_{j} U_{e j}^{2}\right)\left(\sum_{j=1}^{3} U_{e j} T_{e j}^{*}\right)\right] .
\end{gathered}
$$

Here, $m_{\beta \beta}$ is the effective Majorana neutrino mass defined by the neutrino mass eigenvalues $m_{j}$ and the neutrino mixing matrix elements $U_{e j}$ (Horoi and Stoica, 2010):

$$
\left\langle m_{\beta \beta}\right\rangle=\left|\sum_{j} m_{j} U_{e j}^{2}\right| .
$$

The $U$, and $T$ are the $3 \times 3$ block matrices in flavor space, which constitute a generalization of the Pontecorvo-MakiNakagawa-Sakata matrix, namely the $6 \times 6$ unitary neutrino mixing matrix (Štefánik et al., 2015; Šimkovic et al., 2017).

The amplitude of $\lambda$ mechanism is given by (Bhupal Dev et al., 2015) 


$$
\mathcal{A}_{\lambda}=G_{F}^{2} \lambda \sum_{i} U_{e j} T_{e j}^{*} \frac{1}{q},
$$

where $\lambda$ is defined earlier, $G_{F}$ is the Fermi constant for weak interaction, and $q$ is the virtual Majorana neutrino momentum.

The coefficients $C_{I}(I=m m, m \lambda$ and $\lambda \lambda)$ of Eq. 1 are linear combinations of products of nuclear matrix elements and phasespace factors (Šimkovic et al., 2017).

$$
\begin{aligned}
C_{m m} & =g_{A}^{4} M_{\nu}^{2} G_{01}, \\
C_{m \lambda} & =-g_{A}^{4} M_{\nu}\left(M_{2-} G_{03}-M_{1+} G_{04}\right), \\
C_{\lambda \lambda} & =g_{A}^{4}\left(M_{2-}^{2} G_{02}+\frac{1}{9} M_{1+}^{2} G_{011}-\frac{2}{9} M_{1+} M_{2-} G_{010}\right) .
\end{aligned}
$$

Calculated values of phase-space factors $G_{0 i}(i=1,2,3,4,10$ and 11) for different $0 \nu \beta \beta$ decaying nuclei are given in Ref. (Štefánik et al., 2015).

\subsection{Nuclear Matrix Elements for $\lambda$ Mechanism of $0 v \beta \beta$}

Matrix elements required in the expression of $C_{I}$ are (Šimkovic et al., 2017).

$$
\begin{aligned}
M_{\nu} & =M_{G T}-\frac{M_{F}}{g_{A}^{2}}+M_{T}, \\
M_{\nu \omega} & =M_{\omega G T}-\frac{M_{\omega F}}{g_{A}^{2}}+M_{\omega T}, \\
M_{1+} & =M_{q G T}+3 \frac{M_{q F}}{g_{A}^{2}}-6 M_{q T}, \\
M_{2-} & =M_{\nu \omega}-\frac{1}{9} M_{1+\cdot}
\end{aligned}
$$

The $\left(M_{G T, \omega G T, q G T}\right)\left(M_{F, \omega F, q F}\right)$, and $\left(M_{T, \omega T, q T}\right)$ matrix elements of the scalar two-body transition operator $\mathcal{O}_{12}^{\alpha}$ of $0 \nu \beta \beta$ can be expressed as (Brown et al., 2014)

$$
M_{\alpha}=\left\langle f\left|\mathcal{O}_{12}^{\alpha}\right| i\right\rangle
$$

where, $|i\rangle$, and $|f\rangle$ are the initial and the final $0^{+}$ground state (g.s) for $0 \nu \beta \beta$ decay, respectively, and $\alpha=(G T, F, T, v, \omega G T, \omega F$, $\omega T, v \omega, q G T, q F, q T, 1+, 2-), \tau_{-}$is the isospin annihilation operator. The scalar two-particle transition operators $\mathcal{O}_{12}^{\alpha}$ of $0 v \beta \beta$ containing spin and radial neutrino potential operators can be written as

$$
\begin{aligned}
\mathcal{O}_{12}^{G T, \omega G T, q G T} & =\tau_{1-} \tau_{2-}\left(\sigma_{1} . \sigma_{2}\right) H_{G T, \omega G T, q G T}\left(r, E_{k}\right), \\
\mathcal{O}_{12}^{F, \omega F, q F} & =\tau_{1-} \tau_{2-} H_{F, \omega F, q F}\left(r, E_{k}\right), \\
\mathcal{O}_{12}^{T, \omega T, q T} & =\tau_{1-} \tau_{2-} S_{12} H_{T, \omega T, q T}\left(r, E_{k}\right),
\end{aligned}
$$

where, $S_{12}=3\left(\sigma_{1} \cdot \hat{\mathbf{r}}\right)\left(\sigma_{2} \cdot \hat{\mathbf{r}}\right)-\left(\sigma_{1} . \sigma_{2}\right), \mathbf{r}=\mathbf{r}_{1}-\mathbf{r}_{2}$, and $r=|\mathbf{r}|$ is inter nucleon distance of the decaying nucleons. The $E_{k}$ is the energy of the virtual intermediate state $(|k\rangle)$ of $0 \nu \beta \beta$. The intermediate state $|k\rangle$ is achieved when one neutron from the initial state $|i\rangle$ is converted into one proton. Subsequently, from the $|k\rangle$ state, another neutron is converted into another proton to achieve the final state $|f\rangle$ of the $0 v \beta \beta$. For the present manuscript, $|i\rangle$ is the $0^{+}$ g.s. of ${ }^{82} \mathrm{Se},|f\rangle$ is the $0^{+}$g.s. of ${ }^{82} \mathrm{Kr}$, and $|k\rangle$ are all the allowed spin-parity states of intermediate nucleus ${ }^{82} \mathrm{Br}$.

There are two approximations for calculating the NME, one is non-closure approximation and another is the widely used closure approximation. In non-closure approximation, the radial neutrino potential $H_{\alpha}\left(r, E_{k}\right)$ has explicit dependence on energy of the intermediate state $|k\rangle$. In non-closure approximation, the radial neutrino potential for $\lambda$ mechanism of $0 v \beta \beta$ are is given as integral over Majorana neutrino momentum $q$ (Sen'kov and Horoi, 2013):

$$
H_{\alpha}\left(r, E_{k}\right)=\frac{2 R}{\pi} \int_{0}^{\infty} \frac{f_{\alpha}(q, r) q d q}{\left.q+E_{k}-\left(E_{i}+E_{f}\right) / 2\right)}
$$

where $R$ is the radius of the parent nucleus, and the $f_{\alpha}(q, r)$ factor (Appendix B) contains the form factors that incorporates the effects of finite nucleon size (FNS), and higher-order currents (HOC) of nucleons (Šimkovic et al., 1999), which is given in Appendix B of the manuscript. The $E_{i}$ and $E_{f}$ are the g.s. energy of the initial and final nucleus of the $0 \nu \beta \beta$ decay, respectively. The non-closure approximation is computationally very challenging, because in this approximation, the NME has explicit dependence on the energy of large numbers of virtual intermediate state $|k\rangle$ and calculating these states requires enormous computational power. Particularly, for higher mass region isotopes, some of the calculations are still beyond the reach of current generation's high-performance computers. Fortunately, the most of the contributions on NME of $0 \nu \beta \beta$ come from low lying energy states up to $10-12 \mathrm{MeV}$ of the intermediate nucleus (Sen'kov and Horoi, 2013; Sarkar et al., 2020a). Thus, one can replace the effects of $E_{k}$ with a suitable constant energy called closure energy $\langle E\rangle$ without affecting the value of NME too much, and this approximation is known as closure approximation. In this approximation, one assumes (Sen'kov and Horoi, 2013)

$$
\left(E_{k}-\left(E_{i}+E_{f}\right) / 2\right) \rightarrow\langle E\rangle
$$

and the radial neutrino potential operator of Eq. 16 becomes

$$
H_{\alpha}(r)=\frac{2 R}{\pi} \int_{0}^{\infty} \frac{f_{\alpha}(q, r) q d q}{q+\langle E\rangle},
$$

In closure approximation, the $0 \nu \beta \beta$ decay operators defined in Eq. 15 become

$$
\begin{aligned}
& O_{12}^{G T, \omega G T, q G T}=\tau_{1-} \tau_{2-}\left(\sigma_{1} \cdot \sigma_{2}\right) H_{G T, \omega G T, q G T}(r), \\
& O_{12}^{F, \omega F, q F}=\tau_{1-} \tau_{2-} H_{F, \omega F, q F}(r), \\
& O_{12}^{T, \omega T, q T}=\tau_{1-} \tau_{2-} S_{12} H_{T, \omega T, q T}(r) .
\end{aligned}
$$

The closure approximation is widely used in literature as it eliminates the complexity of calculating a large number of virtual intermediate states (Horoi and Stoica, 2010; Sen'kov and Horoi, 2013; Sarkar et al., 2020a). One can find suitable values of $\langle E\rangle$ using the method described in Ref. (Sarkar et al., 2020a), such that using closure approximation, one can get NME near to the nonclosure approximation. 
TABLE 1 | Parameters for the short-range correlation (SRC) parametrization of Eq. 21. Values are taken from Ref. (Horoi and Stoica, 2010).

\begin{tabular}{lccc} 
SRC type & a & b & c \\
\hline Miller-Spencer & 1.10 & 0.68 & 1.00 \\
CD-Bonn & 1.52 & 1.88 & 0.46 \\
AV18 & 1.59 & 1.45 & 0.92
\end{tabular}

In the calculation of the NME of $0 \nu \beta \beta$, it is also necessary to take into account the effects of short-range correlations (SRC). A standard method to include SRC is via a phenomenological Jastrow-like function (Vogel, 2012; Menéndez et al., 2009; Šimkovic et al., 2009). Including SRC effect in the Jastrow approach, one can write the NME of $0 v \beta \beta$ defined in Eq. 14 as (Vogel, 2012)

$$
M_{\alpha}=\left\langle f\left|f_{\text {Jastrow }}(r) O_{12}^{\alpha} f_{\text {Jastrow }}(r)\right| i\right\rangle,
$$

where Jastrow-type SRC function is defined as

$$
f_{\text {Jastrow }}(r)=1-c e^{-a r^{2}}\left(1-b r^{2}\right) .
$$

In literature, three different SRC prametrizationparameterization are used: Miller-Spencer, Charge-Dependent Bonn (CD-Bonn), and Argonne V18 (AV18) to parametrize $a, b$, and $c$ (Horoi and Stoica, 2010). These parameters are chosen in such a way that the two-body wave function of two-body matrix elements (TBME) for $0 \nu \beta \beta$ are still normalized. The parameters $a, b$, and $c$ in different SRC parametrizations are given in Table $\mathbf{1}$.

This approach of using a Jastrow-like function to include the effects of SRC is extensively used in Refs. (Menéndez et al., 2009; Horoi and Stoica, 2010; Neacsu et al., 2012).

\subsection{The Closure Method of Nuclear Matrix Elements Calculation for $0 v \beta \beta$ in ISM}

The $\left(M_{G T, \omega G T, q G T}\right)\left(M_{F, \omega F, q F}\right)$, and $\left(M_{T, \omega T, q T}\right)$ matrix elements of the scalar two-body transition operator $O_{12}^{\alpha}$ of $0 \nu \beta \beta$ can be expressed as the sum over the product of the two-body transition density (TBTD) and anti-symmetric two-body matrix elements $\left(\left\langle k_{1}^{\prime}, k_{2}^{\prime}, J T\left|O_{12}^{\alpha}\right| k_{1}, k_{2}, J T\right\rangle_{A}\right)$ (Brown et al., 2014):

$$
\begin{aligned}
M_{\alpha}^{0 \nu} & =\left\langle f\left|O_{12}^{\alpha}\right| i\right\rangle \\
& =\sum_{J, k_{1}^{\prime} \leq k_{2}^{\prime}, k_{1} \leq k_{2}} \operatorname{TBTD}(f, i, J) \times\left\langle k_{1}^{\prime}, k_{2}^{\prime}, J T\left|O_{12}^{\alpha}\right| k_{1}, k_{2}, J T\right\rangle_{A},
\end{aligned}
$$

where, $\alpha=(F, G T, T, \omega F, \omega G T, \omega T, q F, q G T, q T), J$ is the coupled spin of two decaying neutrons or two final created protons, $\tau_{-}$is the isospin annihilation operator, A denotes that the two-body matrix elements (TBME) (Appendix A) are obtained using anti-symmetric two-nucleon wavefunctions, and $k_{1}$ stands for the set of spherical quantum numbers $\left(n_{1}\right.$; $l_{1} ; j_{1}$ ) (similar definition for $k_{2}, k_{1}{ }^{\prime}, k_{2}{ }^{\prime}$ ). The $|i\rangle$ is $0^{+}$ground state (g.s.) of the parent nucleus, and $|f\rangle$ is the $0^{+}$g.s of the granddaughter nucleus.

The TBTD can be expressed as (Brown et al., 2014)

$$
\operatorname{TBTD}(f, i, J)=\left\langle f\left\|\left[A^{+}\left(k_{1}^{\prime}, k_{2}^{\prime}, J\right) \otimes \tilde{A}\left(k_{1}, k_{2}, J\right)\right]^{(0)}\right\| i\right\rangle,
$$

where,

$$
A^{+}\left(k_{1}^{\prime}, k_{2}^{\prime}, J\right)=\frac{\left[a^{+}\left(k_{1}^{\prime}\right) \otimes a^{+}\left(k_{2}^{\prime}\right)\right]_{M}^{J}}{\sqrt{1+\delta_{k_{1}^{\prime} k_{2}^{\prime}}}},
$$

and

$$
\tilde{A}\left(k_{1}, k_{2}, J\right)=(-1)^{J-M} A^{+}\left(k_{1}, k_{2}, J,-M\right)
$$

are the two particle creation and annihilation operator of rank $J$, respectively. Most of the available public shell model code does not provide the option to calculate TBTD directly. One of the ways is to calculate TBTD in terms of a large number of two nucleon transfer amplitudes (TNA), assuming $0 v \beta \beta$ decay occurs through $(n-2)$ channel (Brown et al., 2014). In $(n-$ 2) channel of $0 \nu \beta \beta$, the TNA are calculated with a large set of intermediate states $|m\rangle$ of the $(n-2)$ nucleons system, where $n$ is the number of nucleons for the parent nucleus. In this approach, the TBTD in terms of TNA is expressed as (Brown et al., 2014)

$$
\operatorname{TBTD}(f, i, J)=\sum_{m} \operatorname{TNA}\left(f, m, k_{1}^{\prime}, k_{2}^{\prime}, J_{m}\right) \operatorname{TNA}\left(i, m, k_{1}, k_{2}, J_{m}\right) \text {, }
$$

where, TNA are given by

$$
\operatorname{TNA}\left(f, m, k_{1}^{\prime}, k_{2}^{\prime}, J_{m}\right)=\frac{\left\langle f\left\|A^{+}\left(k_{1}^{\prime}, k_{2}^{\prime}, J\right)\right\| m\right\rangle}{\sqrt{2 J_{0}+1}} .
$$

Here, $J_{m}$ is the spin of the allowed states $|m\rangle$ of intermediate nuclei. $J_{0}$ is spin of $|i\rangle$ and $|f\rangle . J_{m}=J$ when $J_{0}=0$ (Brown et al., 2014).

\section{RESULTS AND DISCUSSION}

We have used JUN45 effective shell model Hamiltonian (Honma et al., 2009) of $f p g$ model space to calculate the relevant initial, intermediate, and final nuclear states for $0 \nu \beta \beta$ of ${ }^{82} \mathrm{Se}$. In the $f p g$ model space, valence nucleons can occupy the orbitals $0 f_{5 / 2}, 1 p_{3 / 2}, 1 p_{1 / 2}$, and $0 g_{9 / 2}$. For the $0 \nu \beta \beta$ decay of ${ }^{82}$ Se through $(n-2)$ channel, the states of allowed spin-parity of ${ }^{80} \mathrm{Se}$ acts as intermediate states for TNA calculations. The nuclear shell model code KSHELL (Shimizu et al., 2019) was used in the calculation. For comparing some of the TNA values, NushellX@MSU (Brown and Rae, 2014) code was also used. In the present calculation, we have included the first 100 states of different allowed spin-parity of ${ }^{80} \mathrm{Se}$ in calculating the TNA. Earlier, it was found that considering around the first 50 states is enough to get the saturated value of NME, as the most dominating contributions come from the first few initial states (Brown et al., 2014; Sarkar et al., 2020b).

We have adopted the widely used closure approximation with the closure energy $\langle E\rangle=0.5 \mathrm{MeV}$. Earlier studies of Refs. (Sarkar et al., 2020a; Sarkar et al., 2020b) suggested that $\langle E\rangle=0.5 \mathrm{MeV}$ is a suitable value that is close to optimal closure energy and, thus, gives NME near to the NME in the non-closure approximation. 
TABLE 2 | NMEs for $0 \nu \beta \beta$ (light neutrino-exchange and $\lambda$ mechanism) of ${ }^{82} \mathrm{Se}$.

\begin{tabular}{lcccc}
\hline NME Type & \multicolumn{4}{c}{ SRC Type } \\
\cline { 2 - 5 } & None & Miller-Spencer & CD-Bonn & AV18 \\
\hline$M_{F}$ & -0.633 & -0.442 & -0.674 & -0.621 \\
$M_{G T}$ & 3.681 & 2.536 & 3.247 & 3.068 \\
$M_{T}$ & -0.020 & -0.020 & -0.020 & -0.020 \\
$M_{\nu}$ & 3.529 & 2.790 & 3.645 & 3.433 \\
$M_{\omega F}$ & -0.630 & -0.441 & -0.671 & -0.618 \\
$M_{\omega G T}$ & 3.075 & 2.453 & 3.165 & 2.986 \\
$M_{\omega T}$ & -0.020 & -0.020 & -0.020 & -0.020 \\
$M_{\nu \omega}$ & 3.485 & 2.751 & 3.599 & 3.388 \\
$M_{a F}$ & -0.330 & -0.274 & -0.384 & -0.372 \\
$M_{q G T}$ & 11.667 & 10.167 & 12.538 & 12.184 \\
$M_{a T}$ & -0.097 & -0.097 & -0.097 & -0.097 \\
$M_{1+}$ & 11.636 & 10.241 & 12.409 & 12.076 \\
$M_{2-}$ & 2.192 & 1.613 & 2.220 & 2.046
\end{tabular}

The non-closure method can give the exact value of NME, but the present study is beyond the scope of studying it. But, according to earlier results (Sarkar et al., 2020a; Sarkar et al., 2020b), with $\langle E\rangle$ $=0.5 \mathrm{MeV}$, one can get NME in the closure approximation close to the NME in non-closure approximation (within 1\% difference).

Different types of NMEs for light neutrino-exchange and $\lambda$ mechanism of $0 \nu \beta \beta$ for ${ }^{82} \mathrm{Se}$ is shown in Table 2. Here, NMEs are calculated in different SRC parameterization schemes. All standard effects of FNS + HOC are taken care of in all calculations. It is found that the GamowTeller matrix elements dominate over Fermi and tensor type matrix elements. Also, it is found that the $\mathrm{M}_{q G T}$ type matrix element associated with the $\lambda$ mechanism is relatively large as compared to standard light neutrino-exchange Gamow-Teller matrix element $M_{G T}$. This leads to the large value of total NME $M_{1+}$ for $\lambda$ mechanism as compared to total $\mathrm{NME} M_{\nu}$ for light neutrino-exchange mechanism.

This increment of $M_{q G T}$ type of NME, which is obtained through the new revised expression of the nucleon currents of Ref. (Šimkovic et al., 2017), is surprisingly high. It is coming through the new revised expression of the nucleon currents of Ref. (Šimkovic et al., 2017) which includes the higher-order term (pseudoscalar) of the nucleon currents. In our calculation, Eq. 39 is used to calculate $M_{q G T}$ type NME using the revised formalism of nucleon currents of Refs. (Štefánik et al., 2015; Šimkovic et al., 2017).

An old equivalent expression of Eq. 39 is also found in Ref. (Horoi and Neacsu, 2018), which one can write using Eq. (A2c) and Eq. (A4b) of Ref. (Horoi and Neacsu, 2018) as

$$
f_{q G T}(q, r)=\frac{1}{\left(1+\frac{q^{2}}{\Lambda_{A}^{2}}\right)^{4}} q r j_{1}(q r) .
$$

Using this old value of $f_{q G T}(q, r)$, the $M_{q G T}$ type NME will be significantly smaller, as reported earlier.

Here we include the higher-order current effect of pseudoscalar term in Eq. 39 as suggested in Ref. (Šimkovic
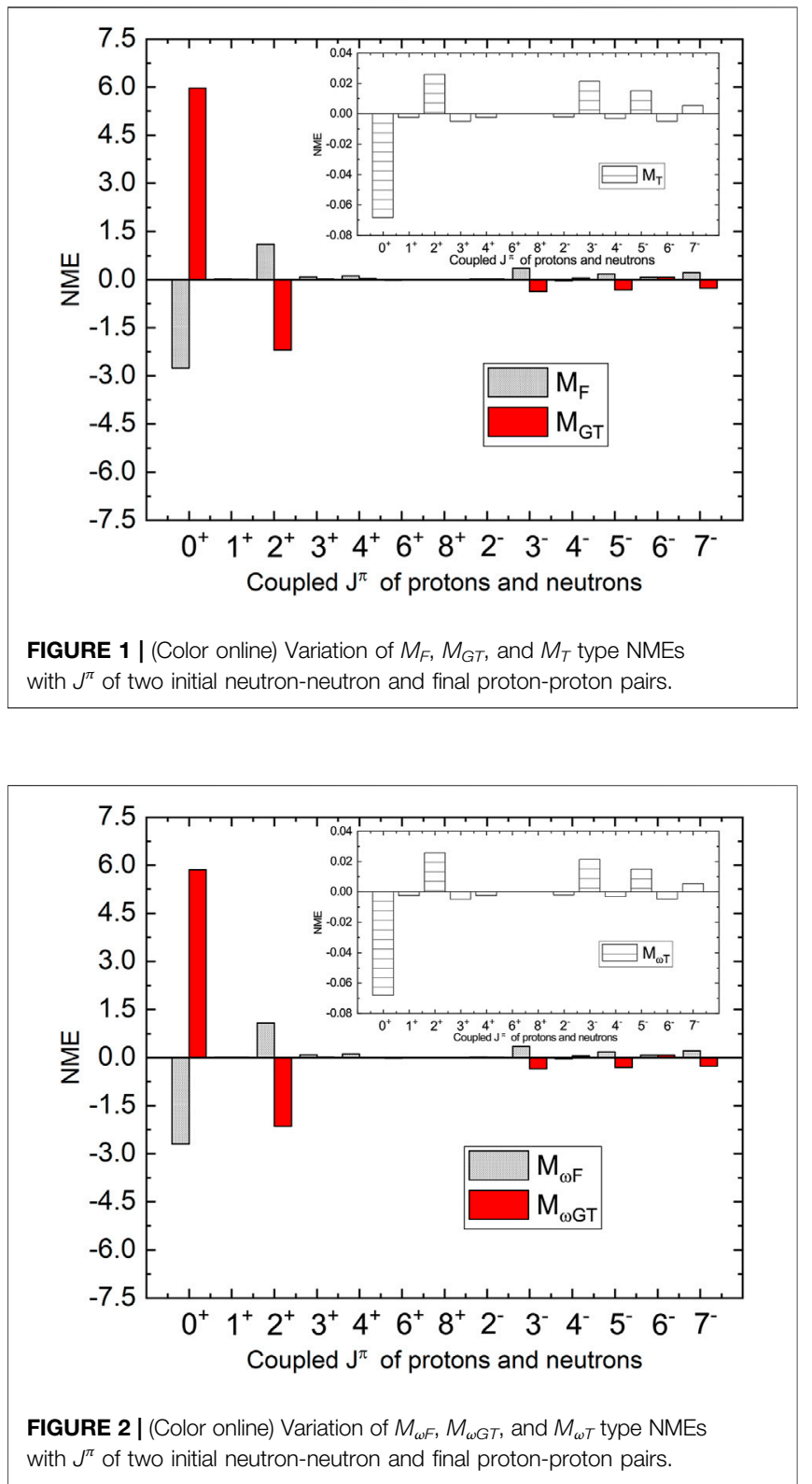

et al., 2017) which is enhancing the $M_{q G T}$ type NME as compared to standard $M_{G T}$ type NME. A similar type of enhancement in $M_{q G T}$ type NME was also found in our earlier study for ${ }^{48} \mathrm{Ca}$ (Sarkar et al., 2020a).

We have also decomposed the NME in terms of coupled spinparity $\left(J^{\pi}\right)$ of two decaying neutrons and two created protons in the decay. Decomposed NME gives us a picture of the role of individual spin-parity on NME. The contribution of NMEs through different $f^{\pi}$ is shown in Figures 1-3 for different types of NME. Figure $\mathbf{1}$ examines the decomposition for $M_{F, G T, T}$ type matrix elements associated with light neutrinoexchange mechanism, where Figures 2, 3 examine the NME as function of $J^{\pi}$ for $M_{\omega F, \omega G T, \omega T}$ and $M_{q F, q G T, q T}$ type NMEs, respectively, for $\lambda$ and interference mechanism. All results are presented for AV18 SRC parameterization. 


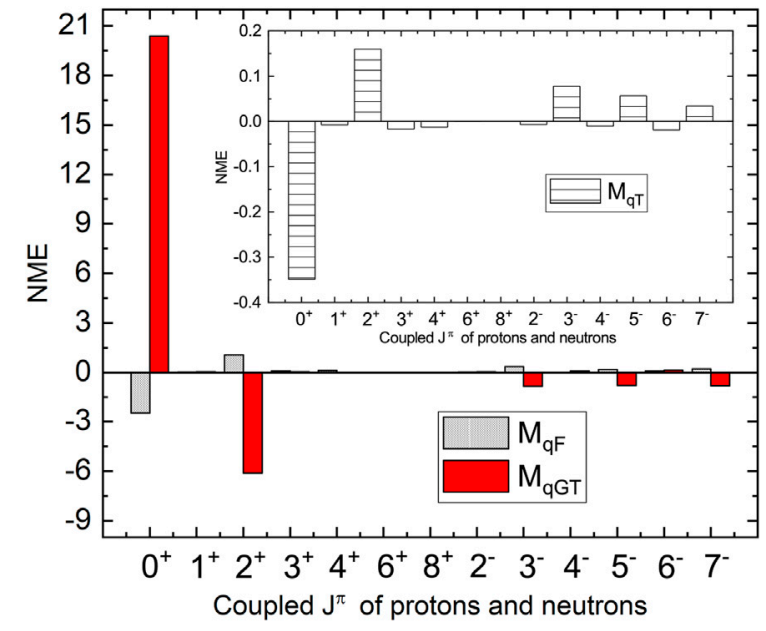

FIGURE 3 | (Color online) Variation of $M_{q F}, M_{q G T}$, and $M_{q T}$ type NMEs with $J^{\pi}$ of two initial neutron-neutron and final proton-proton pairs.

For all types of NMEs, the most dominating contribution comes from $0^{+}$states and $2^{+}$states. The pairing effect is in play for dominating even- $J^{\pi}$ contributions (Brown et al., 2014). The NME from $0^{+}$and $2^{+}$states has opposite signs and, thus, cancel the effects of each other. Other non-negligible contributions come through $4^{+}, 3^{-}, 5^{-}$, and $7^{-}$states.

Now we will discuss how the calculated NMEs will help to determine the bounds on Majorana neutrino mass and various lepton number violating parameters, using the lower limit on the experimental half-life of the decay. The inverse of half-life for $0 \nu \beta \beta$ is given in Eq. 1. It is found that the half-life is influenced by the term $C_{I}(I=m m, m \lambda, \lambda \lambda)$, lepton number violating term $\eta_{v}$ and $\eta_{\lambda}$, which are unknown, and CP-violating phase $\psi$. The $C_{I}$ are defined in Eq 7 and (8), (9), which contains mainly phase space factors and relevant NMEs. To calculate $C_{I}$, we have used the improved values of phase space factors calculated in Ref. (Štefánik et al., 2015), and for the NMEs, we have used the results of Table 2 using ISM.

The results for $C_{I}$ of light neutrino-exchange and $\lambda$ mechanisms of $0 \nu \beta \beta$ decay of ${ }^{82} \mathrm{Se}$ and ${ }^{48} \mathrm{Ca}$ are presented in Table 3. Here, the results for ${ }^{48} \mathrm{Ca}$ are taken from our earlier work using the closure approximation on the $\lambda$ mechanism (Sarkar et al., 2020a). It is found that values of $C_{m m}$ (light neutrinoexchange) and $C_{\lambda \lambda}$ ( $\lambda$ mechanism) are similar in values, which shows the dominance of each of these mechanisms on $0 v \beta \beta$ halflife. The interference term $\left(C_{m \lambda}\right)$ of both the mechanisms are relatively smaller, which shows the less importance of the interference mechanism.

We have also calculated the upper bound on unknown Majorana neutrino mass $\left(m_{\beta \beta}\right)$ and lepton number violating parameter: the right-handed current coupling strength $\eta_{\lambda}$, using the experimental constraint on $T_{1 / 2}^{0 \nu-e x p}$ of Ref. (Arnold et al., 2005) for ${ }^{82} \mathrm{Se}$ and of Ref. (Arnold et al., 2016) for ${ }^{48} \mathrm{Ca}$. The upper limits on $m_{\beta \beta}$ and $\eta_{\lambda}$ are also presented in Table 3 for ${ }^{82} \mathrm{Se}$ and ${ }^{48} \mathrm{Ca}$ when both light neutrino-exchange and $\lambda$ mechanisms co-exist. With the experimental lower limit on $T_{1 / 2}^{0 v-\exp }$, the upper limits on Majorana neutrino mass $\left(m_{\beta \beta}\right)$ are found to be 1.83 and $17.92 \mathrm{eV}$, respectively, for ${ }^{82} \mathrm{Se}$ and ${ }^{48} \mathrm{Ca}$. This difference of $m_{\beta \beta}$ value for ${ }^{82} \mathrm{Se}$ and ${ }^{48} \mathrm{Ca}$ is quite large and also found in earlier work (Šimkovic et al., 2017). With the recent progress and future prospects of new generation experiments, lower limits on $T_{1 / 2}^{0 v-e x p}$ will be gradually improved and thus, will improve the upper limit on $m_{\beta \beta}$ and also reduce the differences for different isotopes.

\section{SUMMARY}

In summary, we have studied how the left-right weak boson exchange $(\lambda)$ mechanism of $0 \nu \beta \beta$ decay is competing with the standard light neutrino-exchange mechanism. Our interest of isotope was one of the prominent $0 \nu \beta \beta$ decaying isotope ${ }^{82} \mathrm{Se}$. Particularly, we have calculated the NMEs for $0 \nu \beta \beta$ of ${ }^{82}$ Se when both standard light neutrino-exchange and $\lambda$ mechanisms coexist. The revised formalism for nucleon currents to include the pseudoscalar term was taken care of. The nuclear shell model framework was used in the calculation, and the widely used closure approximation was adopted with suitable closure energy. Nuclear states of initial, final, and intermediate states are calculated for $f p g$ model space with JUN45 effective shell model Hamiltonian using shell model code KSHELL. These nuclear states are used to calculate TNA, which comes in the expression of NME of $0 \nu \beta \beta$ through $(n-2)$ decay channel. Using the calculated NMEs, we have also calculated the upper bounds on Majorana neutrino mass and lepton number violating parameters.

TABLE 3 | Results for half-life and bounds on neutrino mass and lepton number violating parameters. The $T_{1 / 2}^{0 v-e x p}$ is taken from the experimental lower limit on half-life from Ref. (Arnold et al., 2005) for ${ }^{82} \mathrm{Se}$ and from Ref. (Arnold et al., 2016) for ${ }^{48} \mathrm{Ca}$. All results are for AV18 type SRC parameterizaionparameterization. We have assumed CP conservation $(\psi=0)$. The results are compared with QRPA calculations for $\lambda$ mechanism of Ref. (Šimkovic et al., 2017).

\begin{tabular}{|c|c|c|c|c|}
\hline Quantity & ${ }^{82} \mathrm{Se}$ & $\begin{array}{c}{ }^{82} \text { Se Ref. } \\
\text { Šimkovic } \\
\text { et al. (2017) }\end{array}$ & ${ }^{48} \mathrm{Ca}$ & $\begin{array}{c}{ }^{48} \text { Ca Ref. } \\
\text { Šimkovic } \\
\text { et al. (2017) }\end{array}$ \\
\hline$T_{1 / 2}^{0 v-\exp }$ [Years] & $2.5 \times 10^{23}$ & $2.5 \times 10^{23}$ & $2.0 \times 10^{22}$ & $2.0 \times 10^{22}$ \\
\hline$C_{m m}[\text { Years] }]^{-1}$ & $31.21 \times 10^{-14}$ & $51.3 \times 10^{-14}$ & $4.06 \times 10^{-14}$ & $2.33 \times 10^{-14}$ \\
\hline$C_{m \lambda}[\text { Years }]^{-1}$ & $10.46 \times 10^{-14}$ & $-27.0 \times 10^{-14}$ & $3.37 \times 10^{-14}$ & $-1.04 \times 10^{-14}$ \\
\hline$C_{\lambda \lambda}\left[\right.$ Years] ${ }^{-1}$ & $36.19 \times 10^{-14}$ & $150.0 \times 10^{-14}$ & $5.39 \times 10^{-14}$ & $10.1 \times 10^{-14}$ \\
\hline$m_{\beta \beta}[\mathrm{eV}]$ & 1.83 & 1.43 & 17.92 & 23.7 \\
\hline$\eta_{\lambda}$ & $3.32 \times 10^{-6}$ & $1.63 \times 10^{-6}$ & $30.44 \times 10^{-6}$ & $22.30 \times 10^{-6}$ \\
\hline
\end{tabular}


The results show that particularly $M_{q G T}$ type matrix element of $\lambda$ mechanism is significantly enhanced as compared to standard $M_{G T}$ type NME for the inclusion of the higher-order effect of the pseudoscalar term in the nucleon current. A similar type of enhancement in $M_{q G T}$ type NME was also found in our earlier study for ${ }^{48} \mathrm{Ca}$ (Sarkar et al., 2020a). The dominance of $0^{+}$and $2^{+}$ states of neutron-neutron (proton-proton) pairs were also observed, just like earlier studies.

With the experimental lower limits on the half-life, we have used our calculated NMEs to set the upper bounds on Majorana neutrino mass $\left(m_{\beta \beta}\right)$. The upper limits of values of $m_{\beta \beta}$ are found to be 1.83 and $17.92 \mathrm{eV}$, respectively, for ${ }^{82} \mathrm{Se}$ and ${ }^{48} \mathrm{Ca}$. With the new generation of experiments, the lower limit on half-life will be further improved, and thus we can expect a much more refined upper bound on $m_{\beta \beta}$, which may be below $1 \mathrm{eV}$. Also, the difference for the value of $m_{\beta \beta}$ will be reduced.

The term $C_{I}(I=m m, m \lambda, \lambda \lambda)$, which contains the phase space factors and NMEs, was also evaluated. The $C_{m m}$ for light neutrino exchange and $C_{\lambda \lambda}$ for $\lambda$ mechanism were found to be similar in values, that were larger than the term $C_{m \lambda}$ for the interference of both the mechanisms. This shows the dominance of light neutrino exchange and the $\lambda$ mechanisms over the interference mechanism. The overall dominant effect of light neutrinoexchange mechanism is observed over $\lambda$ mechanism and interference of both the mechanisms for very small values of lepton number violating $\eta_{\lambda}$ parameter.

In the future, it will be interesting to see the competing effect of the $\lambda$ mechanism on the light neutrino-exchange mechanism and also how their contribution on $0 v \beta \beta$ half-life will be evaluated in the current and future generation experiments.

\section{REFERENCES}

Arnold, R., Augier, C., Baker, J., Barabash, A., Broudin, G., Brudanin, V., et al. (2005). First Results of the Search for Neutrinoless Double-Beta Decay with the NEMO 3 Detector. Phys. Rev. Lett. 95, 182302. doi:10.1103/ PhysRevLett.95.182302

Arnold, R., Augier, C., Bakalyarov, A. M., and Baker, J. D. (2016). Measurement of the Double-Beta Decay Half-Life and Search for the Neutrinoless Double-Beta Decay of ${ }^{48} \mathrm{Ca}$ with the NEMO-3 Detector. Phys. Rev. D 93, 112008. doi:10.1103/PhysRevD.93.112008

Arnold, R., Augier, C., Barabash, A. S., Basharina-Freshville, A., Blondel, S., Blot, S., et al. (2020). Search for the Double-Beta Decay of 82 se to the Excited States of $82 \mathrm{kr}$ with Nemo-3. Nucl. Phys. A 996, 121701. doi:10.1016/j.nuclphysa.2020.121701

Avignone, F. T., Elliott, S. R., and Engel, J. (2008). Double Beta Decay, Majorana Neutrinos, and Neutrino Mass. Rev. Mod. Phys. 80, 481-516. doi:10.1103/ RevModPhys.80.481

Barea, J., and Iachello, F. (2009). Neutrinoless Double-Bdecay in the Microscopic Interacting Boson Model. Phys. Rev. C 79, 044301. doi:10.1103/PhysRevC.79.044301

Barea, J., Kotila, J., and Iachello, F. (2012). Limits on Neutrino Masses from Neutrinoless Double- $\beta$ Decay. Phys. Rev. Lett. 109, 042501. doi:10.1103/ PhysRevLett.109.042501

Barry, J., and Rodejohann, W. (2013). Lepton Number and Flavour Violation in Tev-Scale Left-Right Symmetric Theories with Large Left-Right Mixing. J. High Energ. Phys. 2013, 1-45. doi:10.1007/jhep09(2013)153

Bhupal Dev, P. S., Goswami, S., and Mitra, M. (2015). Tev-scale Left-Right Symmetry and Large Mixing Effects in Neutrinoless Double Beta Decay. Phys. Rev. D 91, 113004. doi:10.1103/PhysRevD.91.113004

\section{DATA AVAILABILITY STATEMENT}

The raw data supporting the conclusion of this article will be made available by the authors, without undue reservation.

\section{AUTHOR CONTRIBUTIONS}

The idea of the article was originated by YI. He has also contributed to calculating the nuclear states, interpretation of results, and manuscript writing. SS is responsible for the calculation of the TNA and TBME part of the NME. He has also actively participated in preparing the manuscript. Overall, both the authors have contributed enough in to various stages of preparing the final manuscript.

\section{FUNDING}

YI is grateful for the funding support from JSPS KAKENHI Grant No.17K05440.

\section{ACKNOWLEDGMENTS}

Numerical computation in this work was carried out at the Yukawa Institute Computer Facility. YI acknowledges the Tokyo Institute of Technology for allowing to use of the highperformance computing facility to perform nuclear states calculation using KSHELL. YI is also grateful to Prof. Noritaka Shimizu, CNS, the University of Tokyo, for providing the 2020 version of shell model code KSHELL.

Brown, B. A., Horoi, M., and Sen'kov, R. A. (2014). Nuclear Structure Aspects of Neutrinoless Double- $\beta$ Decay. Phys. Rev. Lett. 113, 262501. doi:10.1103/ PhysRevLett.113.262501

Brown, B. A., and Rae, W. D. M. (2014). The Shell-Model Code NuShellX@MSU. Nucl. Data Sheets 120, 115-118. doi:10.1016/j.nds.2014.07.022

Caurier, E., Menéndez, J., Nowacki, F., and Poves, A. (2008). Influence of Pairing on the Nuclear Matrix Elements of the Neutrinoless Betabeta Decays. Phys. Rev. Lett. 100, 052503. doi:10.1103/PhysRevLett.100.052503

Cirigliano, V., Dekens, W., de Vries, J., Graesser, M. L., Mereghetti, E., Pastore, S., et al. (2019). Renormalized Approach to Neutrinoless Double- $\beta$ Decay. Phys. Rev. C 100, 055504. doi:10.1103/physrevc.100.055504

Deppisch, F. F., Hirsch, M., and Päs, H. (2012). Neutrinoless Double-Beta Decay and Physics beyond the Standard Model. J. Phys. G: Nucl. Part. Phys. 39, 124007. doi:10.1088/0954-3899/39/12/124007

Dolinski, M. J., Poon, A. W. P., and Rodejohann, W. (2019). Neutrinoless DoubleBeta Decay: Status and Prospects. Annu. Rev. Nucl. Part. Sci. 69, 219-251. doi:10.1146/annurev-nucl-101918-023407

Engel, J., and Menéndez, J. (2017). Status and Future of Nuclear Matrix Elements for Neutrinoless Double-Beta Decay: a Review. Rep. Prog. Phys. 80, 046301. doi:10.1088/1361-6633/aa5bc5

Honma, M., Otsuka, T., Mizusaki, T., and Hjorth-Jensen, M. (2009). New Effective Interaction Forf5pg9-Shell Nuclei. Phys. Rev. C 80. doi:10.1103/ physrevc.80.064323

Horoi, M., and Neacsu, A. (2016). Analysis of Mechanisms that Could Contribute to Neutrinoless Double-Beta Decay. Phys. Rev. D 93, 113014. doi:10.1103/ PhysRevD.93.113014

Horoi, M., and Neacsu, A. (2018). Shell Model Study of Using an Effective Field Theory for Disentangling Several Contributions to Neutrinoless Double- $\beta$ Decay. Phys. Rev. C 98, 035502. doi:10.1103/PhysRevC.98.035502 
Horoi, M., and Stoica, S. (2010). Shell Model Analysis of the Neutrinoless Double- $\beta$ Decay of ${ }^{48}$ Ca. Phys. Rev. C 81, 024321. doi:10.1103/PhysRevC.81.024321

Iwata, Y., Shimizu, N., Otsuka, T., Utsuno, Y., Menéndez, J., Honma, M., et al. (2016). Large-Scale Shell-Model Analysis of the Neutrinoless $\beta \beta$ Decay of ${ }^{48} \mathrm{Ca}$. Phys. Rev. Lett. 116, 112502. doi:10.1103/PhysRevLett.116.112502

Menéndez, J., Poves, A., Caurier, E., and Nowacki, F. (2009). Disassembling the Nuclear Matrix Elements of the Neutrinoless $\beta \beta$ Decay. Nucl. Phys. A 818, 139-151. doi:10.1016/j.nuclphysa.2008.12.005

Mohapatra, R. N. (1986). New Contributions to Neutrinoless Double-Beta Decay in Supersymmetric Theories. Phys. Rev. D 34, 3457-3461. doi:10.1103/ PhysRevD.34.3457

Mohapatra, R. N., and Senjanović, G. (1980). Neutrino Mass and Spontaneous Parity Nonconservation. Phys. Rev. Lett. 44, 912-915. doi:10.1103/ PhysRevLett.44.912

Mohapatra, R. N., and Vergados, J. D. (1981). New Contribution to Neutrinoless Double Beta Decay in Gauge Models. Phys. Rev. Lett. 47, 1713-1716. doi:10.1103/PhysRevLett.47.1713

Neacsu, A., Stoica, S., and Horoi, M. (2012). Fast, Efficient Calculations of the TwoBody Matrix Elements of the Transition Operators for Neutrinoless Double- $\beta$ Decay. Phys. Rev. C 86, 067304. doi:10.1103/physrevc.86.067304

Pagnanini, L., Azzolini, O., Beeman, J., Bellini, F., Beretta, M., Biassoni, M., et al. (2019). "Results on Double Beta Decay of ${ }^{82}$ se with CUPID-0 Phase I," in AIP Conference Proceedings (AIP Publishing LLC), 020019. doi:10.1063/1.5130980

Pastore, S., Carlson, J., Cirigliano, V., Dekens, W., Mereghetti, E., and Wiringa, R. B. (2018). Neutrinoless Double- $\beta$ Decay Matrix Elements in Light Nuclei. Phys. Rev. C 97, 014606. doi:10.1103/PhysRevC.97.014606

Rath, P. K., Chandra, R., Chaturvedi, K., Raina, P. K., and Hirsch, J. G. (2010). Uncertainties in Nuclear Transition Matrix Elements for Neutrinoless $\beta \beta$ Decay within the Projected-Hartree-Fock-Bogoliubov Model. Phys. Rev. C 82, 064310. doi:10.1103/PhysRevC.82.064310

Rodejohann, W. (2011). Neutrino-less Double Beta Decay and Particle Physics. Int. J. Mod. Phys. E 20, 1833-1930. doi:10.1142/s0218301311020186

Rodin, V. A., Faessler, A., Šimkovic, F., and Vogel, P. (2006). Assessment of Uncertainties in QRPA 0v $\beta \beta$-decay Nuclear Matrix Elements. Nucl. Phys. A 766, 107-131. doi:10.1016/j.nuclphysa.2005.12.004

Rodríguez, T. R., and Martínez-Pinedo, G. (2010). Energy Density Functional Study of Nuclear Matrix Elements for Neutrinoless $\beta \beta$ Decay. Phys. Rev. Lett. 105, 252503. doi:10.1103/physrevlett.105.252503

Sarkar, S., Iwata, Y., and Raina, P. K. (2020). Nuclear Matrix Elements for the $\lambda$ Mechanism of $0 v \beta \beta$ Decay of ${ }^{48} \mathrm{Ca}$ in the Nuclear Shell-Model: Closure versus Nonclosure Approach. Phys. Rev. C 102, 034317. doi:10.1103/ PhysRevC.102.034317

Sarkar, S., Kumar, P., Jha, K., and Raina, P. K. (2020). Sensitivity of Nuclear Matrix Elements of $0 \nu \beta \beta$ of ${ }^{48} \mathrm{Ca}$ to Different Components of the Two-Nucleon Interaction. Phys. Rev. C 101, 014307. doi:10.1103/PhysRevC.101.014307

Schechter, J., and Valle, J. W. F. (1982). Neutrinoless Double-Bdecay in SU(2)× U(1) Theories. Phys. Rev. D 25, 2951-2954. doi:10.1103/physrevd.25.2951

Sen'kov, R. A., and Horoi, M. (2013). Neutrinoless Double- $\beta$ Decay of ${ }^{48} \mathrm{Ca}$ in the Shell Model: Closure versus Nonclosure Approximation. Phys. Rev. C 88, 064312. doi:10.1103/PhysRevC.88.064312
Sen'kov, R. A., Horoi, M., and Brown, B. A. (2014). Neutrinoless Double- $\beta$ Decay of ${ }^{82} \mathrm{Se}$ in the Shell Model: Beyond the Closure Approximation. Phys. Rev. C 89, 054304. doi:10.1103/PhysRevC.89.054304

Shimizu, N., Mizusaki, T., Utsuno, Y., and Tsunoda, Y. (2019). Thick-restart Block Lanczos Method for Large-Scale Shell-Model Calculations. Comp. Phys. Commun. 244, 372-384. doi:10.1016/j.cpc.2019.06.011

Šimkovic, F., Faessler, A., Müther, H., Rodin, V., and Stauf, M. (2009). 0 $\nu \beta \beta$-decay Nuclear Matrix Elements with Self-Consistent Short-Range Correlation. Phys. Rev. C 79, 055501. doi:10.1103/PhysRevC.79.055501

Šimkovic, F., Pantis, G., Vergados, J. D., and Faessler, A. (1999). Additional Nucleon Current Contributions to Neutrinoless Double $\beta$ Decay. Phys. Rev. C 60, 055502. doi:10.1103/PhysRevC.60.055502

Šimkovic, F., Štefánik, D., and DvornickỲ, R. (2017). The $\lambda$ Mechanism of the

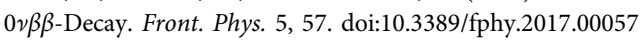

Song, L. S., Yao, J. M., Ring, P., and Meng, J. (2014). Relativistic Description of Nuclear Matrix Elements in Neutrinoless Double- $\beta$ Decay. Phys. Rev. C 90, 054309. doi:10.1103/PhysRevC.90.054309

Štefánik, D., DvornickỲ, R., Šimkovic, F., and Vogel, P. (2015). Reexamining the Light Neutrino Exchange Mechanism of the $0 \nu \beta \beta$ Decay with Left-And RightHanded Leptonic and Hadronic Currents. Phys. Rev. C 92, 055502. doi:10.1103/ PhysRevC.92.055502

Tomoda, T. (1991). Double Beta Decay. Rep. Prog. Phys. 54, 53-126. doi:10.1088/ 0034-4885/54/1/002

Vergados, J. D., Ejiri, H., and Šimkovic, F. (2012). Theory of Neutrinoless DoubleBeta Decay. Rep. Prog. Phys. 75, 106301. doi:10.1088/0034-4885/75/10/106301

Vergados, J. D. (1987). Neutrinoless Double $\beta$-decay without Majorana Neutrinos in Supersymmetric Theories. Phys. Lett. B 184, 55-62. doi:10.1016/03702693(87)90487-4

Vogel, P. (2012). Nuclear Structure and Double Beta Decay. J. Phys. G: Nucl. Part. Phys. 39, 124002. doi:10.1088/0954-3899/39/12/124002

Wang, X. B., Hayes, A. C., Carlson, J., Dong, G. X., Mereghetti, E., Pastore, S., et al. (2019). Comparison between Variational Monte Carlo and Shell Model Calculations of Neutrinoless Double Beta Decay Matrix Elements in Light Nuclei. Phys. Lett. B 798, 134974. doi:10.1016/j.physletb.2019.134974

Conflict of Interest: The authors declare that the research was conducted in the absence of any commercial or financial relationships that could be construed as a potential conflict of interest.

Publisher's Note: All claims expressed in this article are solely those of the authors and do not necessarily represent those of their affiliated organizations, or those of the publisher, the editors, and the reviewers. Any product that may be evaluated in this article, or claim that may be made by its manufacturer, is not guaranteed or endorsed by the publisher.

Copyright $\odot 2021$ Iwata and Sarkar. This is an open-access article distributed under the terms of the Creative Commons Attribution License (CC BY). The use, distribution or reproduction in other forums is permitted, provided the original author(s) and the copyright owner(s) are credited and that the original publication in this journal is cited, in accordance with accepted academic practice. No use, distribution or reproduction is permitted which does not comply with these terms. 


\section{APPENDIX A}

One can write anti-symmetric two-body matrix elements for transition operator $O_{12}^{\alpha}$ of $0 v \beta \beta$ defined in Eq. 22 as

$$
\begin{aligned}
& \left\langle n_{1}^{\prime} l_{1}^{\prime} j_{1}^{\prime}, n_{2}^{\prime} l_{2}^{\prime} j_{2}^{\prime}: J T\left|\tau_{-1} \tau_{-2} O_{12}^{\alpha}\right| n_{1} l_{1} j_{1}, n_{2} l_{2} j_{2}: J T\right\rangle_{A} \\
& =\frac{1}{\sqrt{\left(1+\delta_{j_{1} j_{2}^{\prime}}\right)\left(1+\delta_{j_{1} j_{2}}\right)}} \\
& \left(\left\langle n_{1}^{\prime} l_{1}^{\prime} j_{1}^{\prime}, n_{2}^{\prime} l_{2}^{\prime} j_{2}^{\prime}: J T\left|\tau_{-1} \tau_{-2} O_{12}^{\alpha}\right| n_{1} l_{1} j_{1}, n_{2} l_{2} j_{2}: J T\right\rangle\right. \\
& -(-1)^{j_{1}+j_{2}+J} \\
& \left.\times\left\langle n_{1}^{\prime} l_{1}^{\prime} j_{1}^{\prime}, n_{2}^{\prime} l_{2}^{\prime} j_{2}^{\prime}: J T\left|\tau_{-1} \tau_{-2} O_{12}^{\alpha}\right| n_{2} l_{2} j_{2}, n_{1} l_{1} j_{1}: J T\right\rangle\right),
\end{aligned}
$$

where,

$$
\begin{aligned}
& \left\langle n_{1}^{\prime} l_{1}^{\prime} j_{1}^{\prime}, n_{2}^{\prime} l_{2}^{\prime} j_{2}^{\prime}: J\left|O_{12}^{\alpha}\right| n_{1} l_{1} j_{1}, n_{2} l_{2} j_{2}: J\right\rangle \\
& =\sum_{S^{\prime}, S} \sum_{\lambda^{\prime}, \lambda}\left[\begin{array}{ccc}
l_{1}^{\prime} & \frac{1}{2} & j_{1}^{\prime} \\
& & \\
l_{2}^{\prime} & \frac{1}{2} & j_{2}^{\prime} \\
\lambda^{\prime} & S^{\prime} & J
\end{array}\right]\left[\begin{array}{lll}
l_{1} & \frac{1}{2} & j_{1} \\
l_{2} & \frac{1}{2} & j_{2} \\
\lambda & S & J
\end{array}\right] \\
& \times \sum_{n^{\prime}, l^{\prime}, N^{\prime}, L^{\prime}} \sum_{n, l, N, L} \sum_{\mathcal{J}} \frac{1}{\sqrt{2 S+1}} \frac{1}{\sqrt{2 \mathcal{J}+1}} U\left(L^{\prime}, l^{\prime}, J, S^{\prime}: \lambda^{\prime} \mathcal{J}\right) \\
& \times U(L, l, J, S: \lambda \mathcal{J})\left\langle n^{\prime}, l^{\prime}, N^{\prime}, L^{\prime} \mid n_{1}^{\prime}, l_{1}^{\prime}, n_{2}^{\prime}, l_{2}^{\prime}\right\rangle \\
& \left.\times\left\langle n, l, N, L \mid n_{1}, l_{1}, n_{2}, l_{2}\right\rangle\right\rangle_{\lambda}\left\langle l^{\prime}, S^{\prime}: \mathcal{J}\left\|S_{12}^{\alpha}\right\| l, S: \mathcal{J}\right\rangle \\
& \times\left\langle n^{\prime}, l^{\prime}\left|H_{\alpha}(r)\right| n, l\right\rangle .
\end{aligned}
$$

One can write in terms of $9 \mathrm{j}$ symbol

$$
\begin{aligned}
& {\left[\begin{array}{lll}
l_{1}^{\prime} & \frac{1}{2} & j_{1}^{\prime} \\
l_{2}^{\prime} & \frac{1}{2} & j_{2}^{\prime} \\
\lambda^{\prime} & S^{\prime} & J
\end{array}\right]} \\
& =\sqrt{\left(2 j_{1}^{\prime}+1\right)\left(2 j_{2}^{\prime}+1\right)\left(2 \lambda^{\prime}+1\right)\left(2 S^{\prime}+1\right)} \times\left\{\begin{array}{lll}
l_{1}^{\prime} & \frac{1}{2} & j_{1}^{\prime} \\
& & \\
l_{2}^{\prime} & \frac{1}{2} & j_{2}^{\prime} \\
\lambda^{\prime} & S^{\prime} & J
\end{array}\right\} .
\end{aligned}
$$

In terms of $6 \mathrm{j}$ symbol one can write

$$
\begin{aligned}
U\left(L^{\prime}, l^{\prime}, J, S^{\prime}: \lambda^{\prime} \mathcal{J}\right)= & (-1)^{L^{\prime}+l^{\prime}+S^{\prime}+J} \sqrt{2 \lambda^{\prime}+1} \sqrt{2 \mathcal{J}+1} \\
& \left\{\begin{array}{lll}
L^{\prime} & l^{\prime} & \lambda^{\prime} \\
S^{\prime} & J & \mathcal{J}
\end{array}\right\} .
\end{aligned}
$$

$\left\langle n^{\prime}, l^{\prime}, N^{\prime}, L^{\prime} \mid n_{1}^{\prime}, l_{1}^{\prime}, n_{2}^{\prime}, l_{2}^{\prime}\right\rangle_{\lambda^{\prime}}$ is the harmonic oscillator bracket used to convert the radial integral of neutrino potential from individual coordinate system of nucleons to relative and center of mass coordinate system of the nucleons.

\section{APPENDIX B}

The $f_{\alpha}(q, r)$ factor of Eq. 16 can be written in terms of radial dependence, spherical Bessel function $j_{p}(q r)(p=0,1,2$ and 3), and FNS + HOC coupling form factors in closure approximation as (Šimkovic et al., 2017).

$$
\begin{aligned}
& f_{G T}(q, r)=\frac{j_{0}(q r)}{g_{A}^{2}}\left(g_{A}^{2}\left(q^{2}\right)-\frac{g_{A}\left(q^{2}\right) g_{P}\left(q^{2}\right)}{m_{N}} \frac{q^{2}}{3}\right. \\
& \left.+\frac{g_{P}^{2}\left(q^{2}\right)}{4 m_{N}^{2}} \frac{q^{4}}{3}+\left(2 \frac{g_{M}^{2}\left(q^{2}\right)}{4 m_{N}^{2}} \frac{q^{2}}{3}\right)\right) \\
& f_{F}(q, r)=g_{V}^{2}\left(q^{2}\right) j_{0}(q r) \text {, } \\
& f_{T}(q, r)=\frac{j_{2}(q r)}{g_{A}^{2}}\left(\frac{g_{A}\left(q^{2}\right) g_{P}\left(q^{2}\right)}{m_{N}} \frac{q^{2}}{3}-\frac{g_{P}^{2}\left(q^{2}\right)}{4 m_{N}^{2}} \frac{q^{4}}{3}\right. \\
& \left.+\frac{g_{M}^{2}\left(q^{2}\right)}{4 m_{N}^{2}} \frac{q^{2}}{3}\right) \\
& f_{\omega G T}(q, r)=\frac{q}{(q+\langle E\rangle)} f_{G T}(q, r) \text {, } \\
& f_{\omega F}(q, r)=\frac{q}{(q+\langle E\rangle)} f_{F}(q, r), \\
& f_{\omega T}(q, r)=\frac{q}{(q+\langle E\rangle)} f_{T}(q, r), \\
& f_{q G T}(q, r)=\left(\frac{g_{A}^{2}\left(q^{2}\right)}{g_{A}^{2}} q+3 \frac{g_{P}^{2}\left(q^{2}\right)}{g_{A}^{2}} \frac{q^{5}}{4 m_{N}^{2}}\right. \\
& \left.+\frac{g_{A}\left(q^{2}\right) g_{P}\left(q^{2}\right)}{g_{A}^{2}} \frac{q^{3}}{m_{N}}\right) r j_{1}(q, r), \\
& f_{q F}(q, r)=r g_{V}^{2}\left(q^{2}\right) j_{1}(q r) q \text {, } \\
& f_{q T}(q, r)=\frac{r}{3}\left(\left(\frac{g_{A}^{2}\left(q^{2}\right)}{g_{A}^{2}} q-\frac{g_{P}\left(q^{2}\right) g_{A}\left(q^{2}\right)}{2 g_{A}^{2}} \frac{q^{3}}{m_{N}}\right) j_{1}(q r)\right. \\
& \left.-\left(9 \frac{g_{P}^{2}\left(q^{2}\right)}{2 g_{A}^{2}} \frac{q^{5}}{20 m_{N}^{2}}\left[2 j_{1}(q r) / 3-j_{3}(q r)\right]\right)\right) \text {, }
\end{aligned}
$$

where one can write in dipole approximation (Šimkovic et al., 1999).

$$
\begin{aligned}
g_{V}\left(q^{2}\right) & =\frac{g_{V}}{\left(1+\frac{q^{2}}{M_{V}^{2}}\right)^{2}}, \\
g_{A}\left(q^{2}\right) & =\frac{g_{A}}{\left(1+\frac{q^{2}}{M_{A}^{2}}\right)^{2}}, \\
g_{M}\left(q^{2}\right) & =\left(\mu_{p}-\mu_{n}\right) g_{V}\left(q^{2}\right),
\end{aligned}
$$




$$
g_{P}\left(q^{2}\right)=\frac{2 m_{p} g_{A}\left(q^{2}\right)}{\left(q^{2}+m_{\pi}^{2}\right)}\left(1-\frac{m_{\pi}^{2}}{M_{A}^{2}}\right) .
$$

$\mu_{p}-\mu_{n}=4.7, M_{V}=850 \mathrm{MeV}, M_{A}=1,086 \mathrm{MeV} m_{p}$ and $m_{\pi}$ are the mass of protons and pions (Sen'kov and Horoi, 2013).
In the present calculation, vector constant $g_{V}=1.0$ and bare axial-vector constant $g_{A}=1.27$ (Sarkar et al., 2020b) are used. Both the pseudo scalar and weak magnetism terms of the nucleon currents are included in $f_{G T, T, \omega G T, \omega T}(q, r)$ factors whereas pseudo scalar term is included in $f_{q G T, q T}(q, r)$ factors (Šimkovic et al., 2017). 